\title{
Comparison of different surgical approaches for anterior mediastinal tumor
}

\author{
Yong Mao" ${ }^{1 \#}$ Yuting $\mathrm{Lan}^{2,3 \#}$, Fei Cui ${ }^{2 \#}$, Hongsheng Deng ${ }^{2}$, Yaoliang Zhang ${ }^{4}, \mathrm{Xi} \mathrm{Wu}^{4}$, Wenhua Liang ${ }^{2}$, \\ Jun Liu ${ }^{2}$, Hengrui Liang ${ }^{2}$, Jianxing $\mathrm{He}^{2}$
}

${ }^{1}$ Department of Cardiothoracic Surgery, Ningbo First Hospital, Ningbo, China; ${ }^{2}$ Department of Thoracic Surgery and Oncology, the First Affiliated Hospital of Guangzhou Medical University, State Key Laboratory of Respiratory Disease, National Clinical Research Center for Respiratory Disease, Guangzhou Institute of Respiratory Health, Guangzhou, China; ${ }^{3}$ Mental Health College, Guangzhou Medical University, Guangzhou, China; ${ }^{4}$ Department of Anesthesia, the First Affiliated Hospital of Guangzhou Medical University, Guangzhou, China

Contributions: (I) Conception and design: Y Mao, H Liang, Y Lan; (II) Administrative support: J He; (III) Provision of study materials or patients: Y Mao, H Liang, H Deng; (IV) Collection and assembly of data: H Liang, Y Lan; (V) Data analysis and interpretation: Y Mao, H Liang, Y Lan; (VI) Manuscript writing: All authors; (VII) Final approval of manuscript: All authors.

"These authors contributed equally to this work.

Correspondence to: Jun Liu, MD; Hengrui Liang, MD; Jianxing He, MD. Department of Thoracic Surgery and Oncology, the First Affiliated Hospital of Guangzhou Medical University, State Key Laboratory of Respiratory Disease, National Clinical Research Center for Respiratory Disease,Guangzhou Institute of Respiratory Health, Guangzhou, China. Email: liujun9707@sina.com; Hengrui_Liang@163.com; drjianxing.he@gmail.com.

Background: Different video-assisted thoracoscopic surgery (VATS) approaches may related to heterogeneous clinical outcomes in anterior mediastinal tumor surgery. Herein, we assessed the comparison between the subxiphoid and intercostal approach, and also compare the left versus the right incision in the intercostal approach for anterior mediastinal tumor patients.

Methods: Clinical data of patients receiving thoracoscopic anterior mediastinal tumor resection were retrospectively collected. Patients were divided into two groups according to the approaches: subxiphoid and the intercostal group. The intercostal group was further subdivided into two groups according to different sides: left and right incision group. Intraoperative and postoperative variables were compared between subgroups.

Results: A total of 238 patients were consecutively included in this analysis; 198 (83.2\%) patients received intercostal procedure and $40(16.8 \%)$ patients received subxiphoid approach. After 1:1 propensity score matching, all baseline characters were well balanced between intercostal and subxiphoid approach, left and right intercostal approach. The visual analogue scale (VAS) pain score was lower in patients underwent subxiphoid approach than intercostal group at first post-operative evaluation in 12-24 h (4.36 vs. 2.23; $\mathrm{P}=0.03)$. According to left and right approach, postoperative drainage time (1.9 vs. 1.2 days, $\mathrm{P}=0.016)$, postoperative drainage volume (312.1 vs. $193.9 \mathrm{~mL}, \mathrm{P}=0.041)$ and hospitalization time (5.3 vs. 4.1 days, $\mathrm{P}=0.043$ ) were significantly increased in the left thoracic approach group compared with the right thoracic approach.

Conclusions: Subxiphoid approach is associated with less pain compared with intercostal approach. The right intercostal thoracic approach may offer better clinical effect of short-term postoperative recovery.

Keywords: Mediastinal tumor; intercostal approach; subxiphoid approach

Submitted Jan 08, 2020. Accepted for publication May 29, 2020.

doi: $10.21037 /$ jtd-20-266

View this article at: http://dx.doi.org/10.21037/jtd-20-266 


\section{Introduction}

Complete resection is essential for mediastinal tumor to achieve satisfactory short and long-term survival (1). Currently, standard surgical methods include sternotomy and video-assisted thoracoscopic surgery (VATS) (2). Median sternotomy has been recognized a more invasive operation, with increased surgical trauma and postoperative complications, affecting postoperative recovery (3). VATS is less invasive and has been shown to be a safe way to treat mediastinal tumors (4).

Subxiphoid and intercostal approaches are the main approaches for anterior mediastinal tumor resection under thoracoscopic in clinical practice, which can reduce postoperative pain and achieve a good therapeutic effect with high safety (5-7). The subxiphoid approach avoids chronic postoperative incisional pain and chest cavities. No requirement for changes in the body position, good cosmetic appearance also make it as an ideal approach for thymectomy. However, limitations of the subxiphoid approach like increasing surgery difficulty also make it as not a good candidate.

In the intercostal approach setting, the right thoracic approach is commonly used because of its high level of safety, but a number of literatures have proved that it is feasible and safe to remove the left and middle mediastinal tumor through left lateral intercostal approach (8-11). This study compared the short-term outcomes of the subxiphoid approach and the intercostal approach. The left intercostal approach and the right intercostal approach was also compared, as to explore the feasibility and safety of different approaches for the anterior mediastinal tumor resection. We present the following article in accordance with the STROBE reporting checklist (available at http://dx.doi. org/10.21037/jtd-20-266).

\section{Methods}

\section{Study design and patient inclusion}

The study was written according to The Strengthening the Reporting of Observational Studies in Epidemiology (STROBE) Statement (12). Perioperative data of all patients with mediastinal tumor admitted to the first affiliated hospital of Guangzhou Medical University from December 2015 to October 2019 were identified and collected through electronic medical records. This study was conducted in accordance with the Declaration of Helsinki (as revised in 2013) and the Harmonized
Tripartite Guideline for Good Clinical Practice from the International Conference on Harmonization. The study protocol and methods were reviewed by the institutional ethics committee of hospital (ID Number: 2019-SVA). Computed tomography (CT) scans of all patients were performed to confirm the size and position of lesions by two independent radiologists before any procedure. Exclusive criteria: cases that underwent median sternotomy, cases that received bilateral surgery to both sides, malignant cases with obvious invasion to the surrounding organs. Only anterior mediastinal abnormalities were included in the subxiphoid approach surgery. Prior to the surgery, anesthetists visited each patient and discussed with surgeons to judge who could be potentially received subxiphoid surgery. The we explain the features of each technique to patients and the patients would make the decision. Informed and written consents for each patient were obtained. The study design flow chart was summarized in Figure 1.

\section{Surgical approaches}

\section{Intercostal approach}

Patients are placed in left or right lateral positions depending on the incision approach. A $2-3 \mathrm{~cm}$ incision was created initially in the fourth or fifth intercostal space at the anterior axillary line. If the target lesion was located above the confluence of brachiocephalic vein and superior vena cava, or extended thymectomy was needed, an incision on the fourth intercostal space was preferred over the fifth. Two ports were created, one $30-\mathrm{mm}$ port is for surgical approach and a 30 angled camera was placed in the lower lateral $15-\mathrm{mm}$ port (13). All specimens were safely removed via a specimen bag by enlarging the upper port. Any bleeding or air leak was managed by reinforcement sutures using 4/0 PROLENE (Ethicon, Somerville, NJ) or application of sealants such as Biopaper (Datsing BioTech Co. Ltd., Beijing, China). After the surgery, the closed thoracic drainage tube was placed through the observation hole, and the chest-X-ray examination showed no obvious pleural effusion or pneumatosis. Chest drain was removed when the drainage was less than $200 \mathrm{~mL} /$ day. The bedside chest-X-ray could be performed at the same time after the chest tube was removed, and food was resumed after the bowel sound returned to 5 times per minute without nausea or vomiting (14). Patients who were able to mobilize independently and appeared normal on chest radiography were discharged after chest tube removal. 


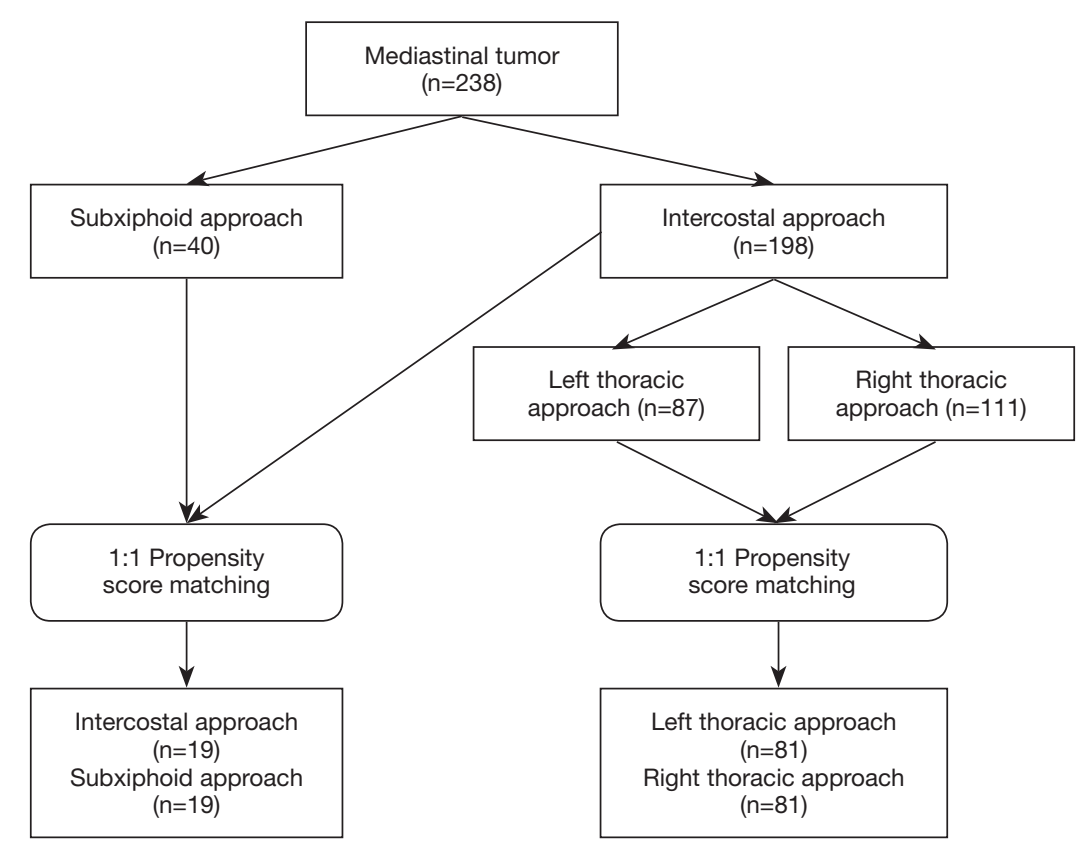

Figure 1 Schema of patient grouping and matching.

\section{Subxiphoid approach}

The surgical process via mediastinal approach have been already reported elsewhere (8). A 3-cm incision was made $2 \mathrm{~cm}$ below the lower edge of the xiphoid for setting the thoracoscope. Two $5 \mathrm{~mm}$ extra pleural thoracic ports were created at the midclavicular line intersecting with the bilateral costal arch to introduce a thoracoscopic grasping forceps and a harmonic scalpel. A pneumomediastinum was created by an $8 \mathrm{cmH}_{2} \mathrm{O}$ positive pressure carbon dioxide $\left(\mathrm{CO}_{2}\right)$ insufflation to enlarge the retrosternal space and facilitate dissection of the tumor. Both the right-sided and left-sided mediastinal pleura were opened. The incision in the chest wall could cause collapse of the lungs. The tumor and surrounding tissue were resected and removed through the subxiphoid port. Finally, the air in the chest cavities was evacuated by inflating the lungs. A drainage tube was inserted into the mediastinum through the subxiphoid incision. If necessary, the process was changed from the subxiphoid approach to the transthoracic approach or open thoracotomy.

\section{Data collection and statistical analysis}

Baseline characteristics were extracted to observe age, gender, body mass index (BMI), forced vital capacity (FVC), forced expiratory volume in one second (FEV1), comorbidity, ASA status, tumor location, tumor size, incision side and other indicators of the experimental group and control group.

The propensity-score matching (PSM) generated from the logistic regression were performed to minimize the differences in confounding variables and facilitate matching patients in the two treatment groups ( $\mathrm{R}$ software version 2.15.1, https://cran.r-project.org/). Variables that could influence the outcomes of treatment were used to generate a propensity score, including age, BMI, tumor length, gender, tumor position, ASA status and number of comorbidities. Patients were 1:1 matched on the basis of PSM using the nearest-neighbor method on the logit scale. The caliper was set at 0.01. After PSM, standardized mean differences (SMD) before and after PSM were calculated. Confounding variables was considered comparable when SMD below 0.10 .

Collecting perioperative indicators, compare groups of patients with operation time, bleeding, conversion to open rate, the morbidity and mortality of 30 days, chest tube duration and volume, length of hospital stay, postoperative blood coagulation function and arterial blood gas index, the safety and recovery, etc.

Continuous data are presented as mean and standard deviation and were analyzed with 2-sample Student $t$-tests for independent data. Categorical variables were used for patient counts and percentages, comparing using the 
Table 1 Demographic characters of patients with subxiphoid approach and intercostal approach before and after 1:1 propensity score matching

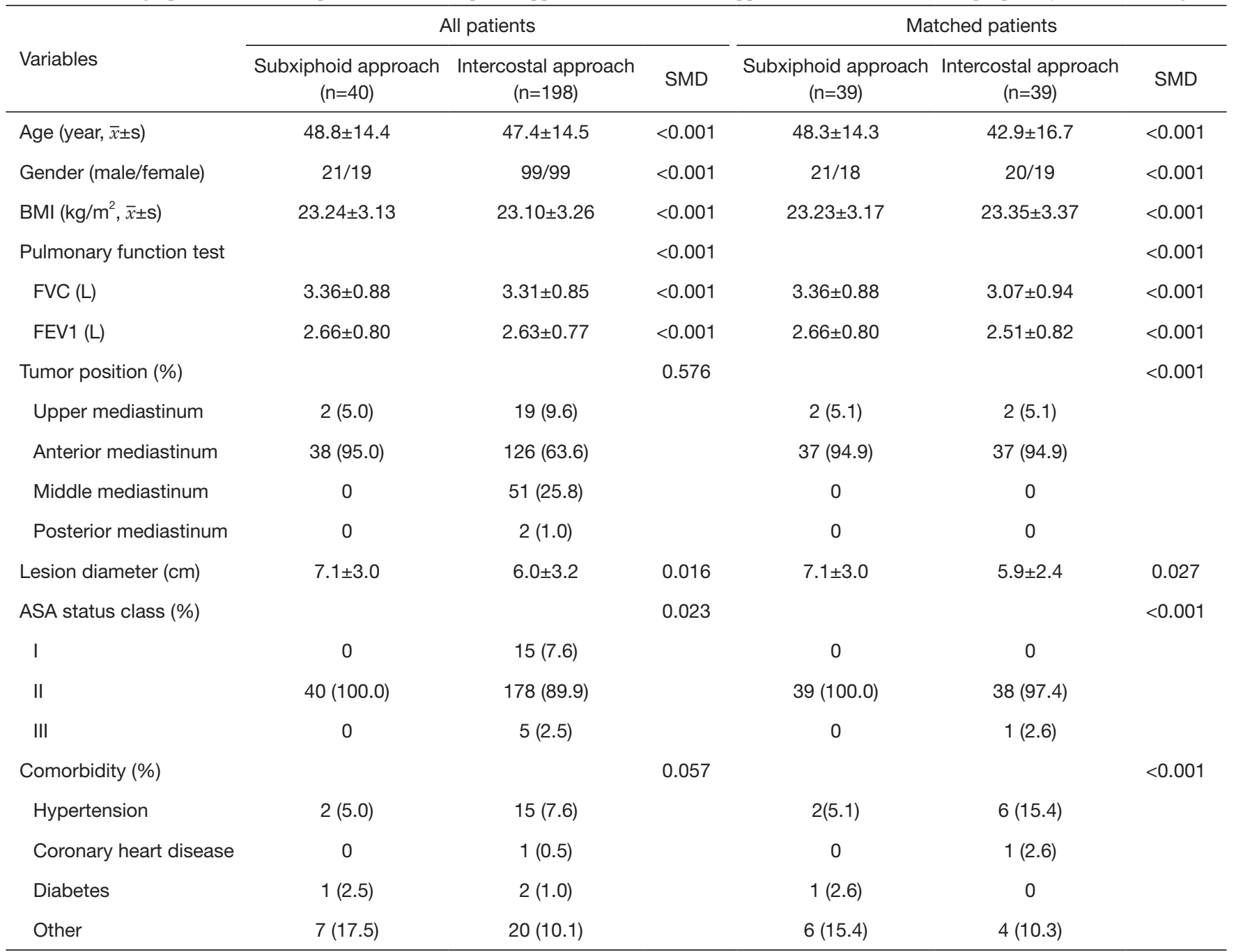

BMI, body mass index; FVC, forced vital capacity; FEV1, forced expiratory volume in 1 second; ASA, American Society of Anesthesiologists; $\mathrm{SD}$, standard deviation.

$\chi^{2}$ or Fisher exact test. All statistical tests were 2-sided, and $\mathrm{P}<0.05$ was considered statistically significant. SPSS software (SPSS version 25.0; IBM Corp, Armonk, NY) was used for all statistical evaluations.

\section{Results}

\section{Baseline data}

A total of 238 patients between December 2015 and October 2019 were consecutively included in this analysis, of which $198(83.2 \%)$ patients received intercostal procedure and $40(16.8 \%)$ patients received subxiphoid approach anterior mediastinal tumor resection (Figure 1). After 1:1 PSM, 19 pairs of patients were collected to evaluate between intercostal and subxiphoid approach, and 81 pairs of patients were collected to compared left and right approach. The baseline characters were well balanced in two groups (Table 1).

Intercostal approaches were divided into the left (87 patients) and the right (111 patients) thoracic approach groups. After 1:1 PSM, 162 cases included in the further analysis, tumor location (anterior), age, gender, BMI, tumor length, diameter and location, preoperative detection of lung function, etc., between the two groups showed no statistical difference $(\mathrm{P}>0.05)$ (Table 2). 
Table 2 Demographic characters of patients with left thoracic approach and right thoracic approach after 1:1 propensity score matching

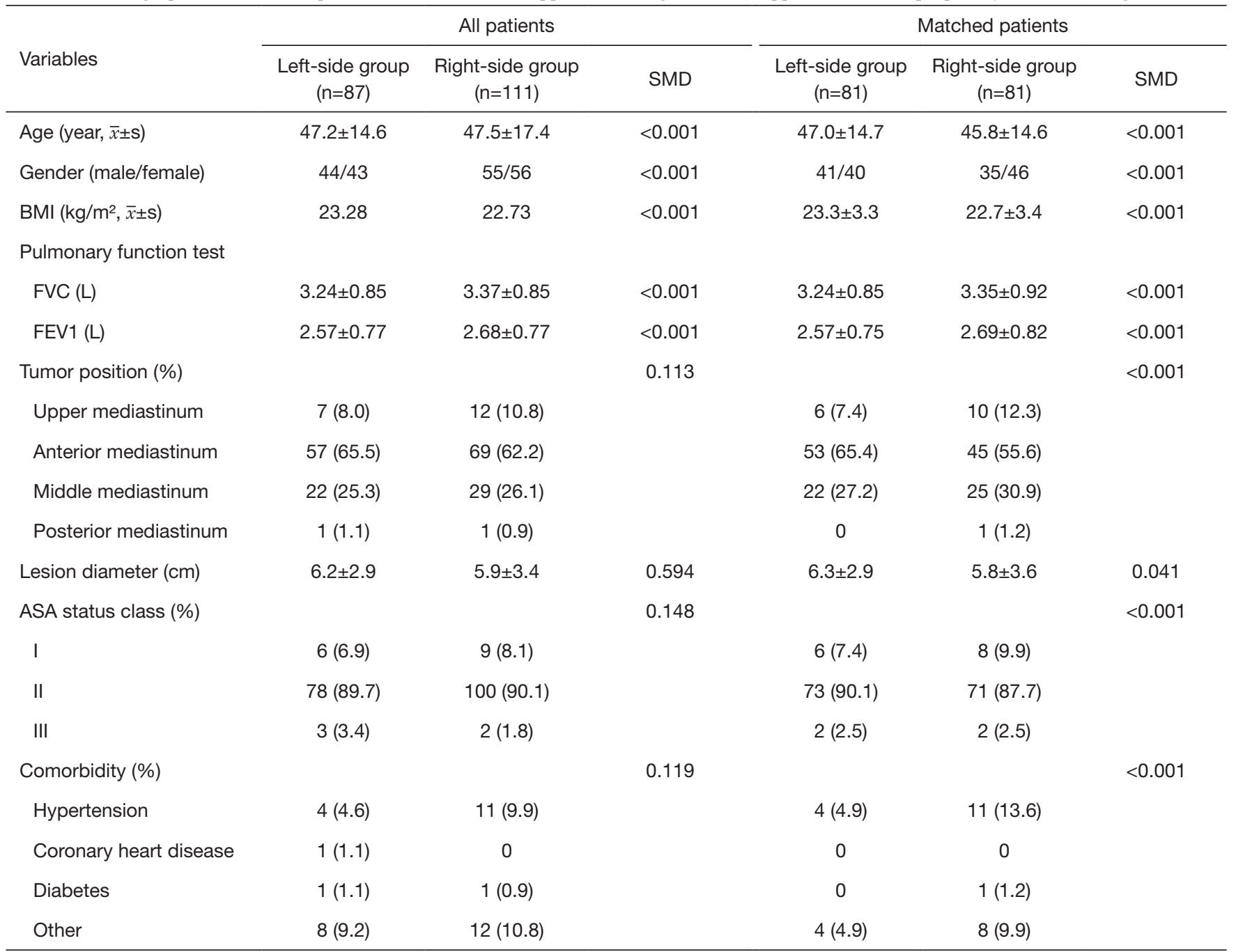

BMI, body mass index; FVC, forced vital capacity; FEV1, forced expiratory volume in 1 second; ASA, American Society of Anesthesiologists; $\mathrm{SD}$, standard deviation.

\section{Intra-operative outcomes}

\section{Intercostal $v s$. subxiphoid approach}

No surgery related death or conversion to open in both groups. The operation time of the intercostal group showed not significantly different (128 vs. $148 \mathrm{~min}, \mathrm{P}=0.173$ ), comparing with the subxiphoid group. The blood loss between two groups also had no significant difference (36 vs. $77 \mathrm{~mL} ; \mathrm{P}=0.246)$.

\section{Left $v$ s. right side}

No damage of main vessels or arteries in two groups. Operation time (108 vs. $102 \mathrm{~min} ; \mathrm{P}=0.530$ ) was comparable in left and right side. Though bleeding amount is more in left side ( 73 vs. $38 \mathrm{~mL} ; \mathrm{P}=0.236$ ), it was no statistical significance.

\section{Post-operative outcomes}

\section{Intercostal $v$ s. subxiphoid approach}

There were no statistically significant differences in drainage time, the total amount of drainage and hospitalization time between the two groups (Table 3). The visual analogue scale (VAS) pain score was lower in patients underwent subxiphoid approach than intercostal group at first post-operative evaluation in $12-24 \mathrm{~h}$ (4.36 vs. 2.23; 
Table 3 Postoperative recovery of patients with subxiphoid approach and intercostal approach

\begin{tabular}{lccc}
\hline Variables & Subxiphoid approach $(\mathrm{n}=19)$ & Intercostal approach $(\mathrm{n}=19)$ & $\mathrm{P}$ value \\
\hline Chest tube duration (day) & $1.9 \pm 1.4$ & $1.7 \pm 2.5$ & 0.738 \\
Drainage of the total $(\mathrm{mL})$ & $360.3 \pm 410.3$ & $255.6 \pm 295.4$ & 0.209 \\
Total length of stay (day) & $3.3 \pm 2.6$ & $5.4 \pm 3.2$ & 0.077 \\
\hline
\end{tabular}

Table 4 Postoperative recovery of patients with left thoracic approach and right thoracic approach

\begin{tabular}{lccc}
\hline Variables & Left-side group $(\mathrm{n}=81)$ & Right-side group $(\mathrm{n}=81)$ & $\mathrm{P}$ value \\
\hline Chest tube duration (day) & $1.9 \pm 2.3$ & $1.2 \pm 1.2$ & 0.016 \\
Drainage of the total $(\mathrm{mL})$ & $312.1 \pm 462.4$ & $193.9 \pm 231.3$ & 0.041 \\
Total length of stay (day) & $5.3 \pm 3.4$ & $4.1 \pm 3.6$ & 0.043 \\
\hline
\end{tabular}

$\mathrm{P}=0.03)$.

\section{Left $v s$. right side}

In comparing the intercostal left to the right thoracic approach, postoperative drainage time (1.9 vs. 1.2 days, $\mathrm{P}=0.016)$, postoperative drainage volume $(312.1$ vs. $193.9 \mathrm{~mL}$, $\mathrm{P}=0.041)$ and hospitalization time (5.3 vs. 4.1 days, $\mathrm{P}=0.043$ ) were significantly increased in the left thoracic approach group compared with the left thoracic approach group (Table 4). The VAS pain score was similar in patients underwent right and left approach at first post-operative evaluation in 12-24 h (4.38 vs. $4.33 ; \mathrm{P}=0.63)$.

\section{Post-operative coagulation function and arterial blood gas}

As for the coagulation function (Table 5), post-operative D-dimer was significant higher in subxiphoid than that was in intercostal approach $(1,383.8 v s .480 .0 \mu \mathrm{g} / \mathrm{L} ; \mathrm{P}=0.001)$. There was no difference between left and right thoracic approach.

As for the arterial blood gas function (Tables 5,6), there was no difference between intercostal and subxiphoid approach. Post-operative $\mathrm{PaO}_{2}$ was significant higher in right side thoracic approach than that was in left side (99.8 vs. $130.7 \mathrm{mmHg}$; $\mathrm{P}=0.001$ ).

\section{Discussion}

Median sternotomy is still the accepted gold standard for mediastinal tumors (9). However, with the development of thoracoscopic assisted minimally invasive surgery, traditional sternal surgery has been characterized by large wounds, large intraoperative blood loss, increased postoperative pain and infection, thus affecting postoperative recovery quality and long-term quality of life $(10,11,15)$. VATS as a minimally invasive technique for mediastinal disease has been widely accepted $(16,17)$.

The subxiphoid procedure has been introduced over 40 years in anterior mediastinal tumors resection (18). Because of less intercostal nerve located, the introduction of the subxiphoid pathway to the field of VATS was aimed at reducing postoperative pain. On the one hand, subxiphoid approach as an ideal approach for thymectomy has radical procedure, cost saving, approach both chest cavities and avoid chronic postoperative incisional pain. A recent investigation also have shown that pain score on the first day after surgery and before discharge in the subxiphoid uniportal VATS group was better than that in the intercostal uniportal VATS group (19). On the other hand, compared with intercostal approach, subxiphoid approach have overt benefits for better cosmetic appearance with less visible chest scars. A published results also indicated that: the subxiphoid approach patients reported higher cosmetic scores than the lateral approach group in terms of cosmetic satisfaction (20). However, subxiphoid approach also has limitations, the intraoperative bleeding is an intractable problem because haemostasis, clamping and suturing is difficult to perform in the deep area when using subxiphoid approach (19). Also, it is not a good candidate when the patients' BMI $>30$ or patients with cardiac disease. Nevertheless, intercostal thoracoscopic surgery has distinct disadvantages including difficulty in identifying the 
Table 5 Post-operative index comparison between subxiphoid approach and intercostal approach

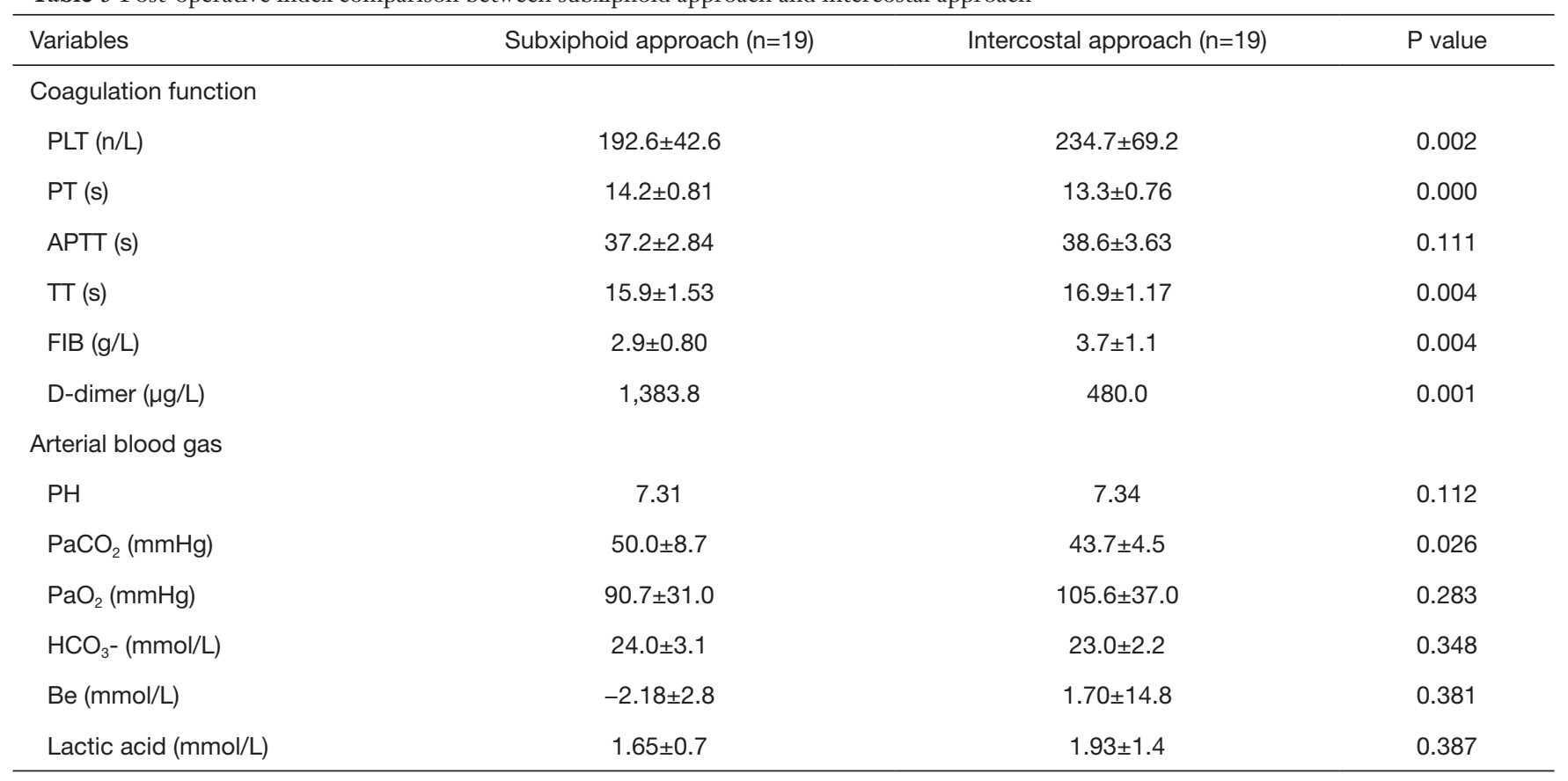

Table 6 Post-operative index comparison between left thoracic approach and right thoracic approach

\begin{tabular}{|c|c|c|c|}
\hline Variables & Left-side group $(n=81)$ & Right-side group $(\mathrm{n}=81)$ & $P$ value \\
\hline PLT (n/L) & $245.8 \pm 66.4$ & $226.2 \pm 52.3$ & 0.039 \\
\hline PT (s) & $13.3 \pm 0.67$ & $13.3 \pm 0.68$ & 0.972 \\
\hline APTT (s) & $38.4 \pm 3.5$ & $38.2 \pm 4.3$ & 0.744 \\
\hline FIB (g/L) & $3.4 \pm 1.0$ & $3.7 \pm 4.1$ & 0.469 \\
\hline D-dimer ( $\mu \mathrm{g} / \mathrm{L})$ & 391.6 & 344.3 & 0.498 \\
\hline \multicolumn{4}{|l|}{ Arterial blood gas } \\
\hline $\mathrm{PH}$ & 7.35 & 7.35 & 0.847 \\
\hline $\mathrm{HCO}_{3}-(\mathrm{mmol} / \mathrm{L})$ & 22.9 & 23.0 & 0.804 \\
\hline $\mathrm{Be}(\mathrm{mmol} / \mathrm{L})$ & -2.2 & 0.15 & 0.283 \\
\hline Lactic acid (mmol/L) & $1.83 \pm 1.0$ & $1.43 \pm 0.72$ & 0.160 \\
\hline
\end{tabular}

PLT, platelets; PT, prothrombin time; APTT, activated partial thromboplastin time; TT, thrombin time; FIB, fibrinogen. 
contralateral phrenic nerve and postoperative pain due to intercostal nerve injury (20). Intercostal nerves is nerves that supply the muscles between the ribs. Due to the proximity of anatomical location, intercostal approach can easily damage the intercostal nerves, leading to the development of intercostal neuralgia, which can manifest as numbness, itchiness, or intermittent and spasmodic pain. However, Intercostal approach surely has advantages of smaller wound surface, easier preoperative preparation and shorter operation time, enabling patients to get better oxygenation during the operation and more advantageous in the quality of postoperative recovery $(21,22)$.

Under the thoracoscopic surgery for mediastinal lesions, the right thoracic approach is away from heart, with high safety, making it widely used for surgery. But for the slant on the left side of the lesion, the right thoracic approach increased the difficulty of mediastinal operation, so under this situation left chest approach was a choice. The specific clinical efficacy of two paths remains controversial within scholars (23). Comparative analysis in our study of the clinical results indicated the postoperative drainage time, drainage volume and hospital stay were benefited in the right thoracic approach group. The less drainage time and hospital stay, the less chance to have postoperative infection, which is conducive to patients' rehabilitation and benefit to their lung function. Perioperative blood coagulation function and other indexes of arterial blood gas showed no significant difference. Thoracoscopic mediastinal tumor resection through the right chest approach is more beneficial to the postoperative recovery of patients.

Compared with the left thoracic approach, the right thoracic space is relatively larger, which also avoids blocking of the pericardium as well as the occlusion of the aortic arch and left ventricle on the surgical field of vision. In particular, it can better avoid the damage of blood vessels and nerves when dealing with the dorsal lesions (11). In this study, there was no significant difference between the left thoracic group and the right thoracic group in the amount of intraoperative blood loss. However, due to the location of human anatomy, the difficulty of the left chest approach operation was relatively greater than that of the right chest approach. The left thoracic approach needs to avoids the anatomic structures such as the aortic arch and its branches. In addition, according to a retrospective study, dissection rates of upper mediastinal lymph node and subcarinal lymph node are lower than that in the right thoracic approach (24), thus the operation time is longer in left approach group.
The postoperative drainage time, total amount and postoperative hospitalization time of patients with left thoracic approach were significantly higher than those with right thoracic approach, which was not conducive to the postoperative recovery of patients (25-27).

The study also has its limitations. Firstly, the study compared approaches of subxiphoid and intercostal approaches, left thoracic approach and right thoracic approach on the indicators during the operation process of thoracoscopic mediastinal tumor resection, as well as the short-term drainage volume and length of hospitalization. However, no follow-up was conducted, so it was not clear whether these thoracoscopic approaches had any effect on the long-term quality of life. Further, we did not answer the question whether different approaches would affect relapse time and long-term survival outcomes. Secondly, studies have shown that the right thoracic approach is beneficial for short-term postoperative recovery (drainage time and total amount, and hospitalization time is less), but the specific mechanism is not clear, the analysis results cannot be clearly explained, and the evidence is insufficient. Third, this study lies in that the retrospective nature introduced biases including surgeons' preference and learning curve for VATS, within which only the measured part was addressed by propensity score matching. Fourth, bilateral intercostal approach is needed for patient having myasthenia gravis regardless of with or without the mediastinal tumors, however, in order to keep the homogeneity of the study, we excluded these cases. Last, retrospective dataset might be related to recalling bias, which may affect the accuracy of this analysis.

\section{Conclusions}

Subxiphoid approach is associated with less pain compared with intercostal approach. The right intercostal thoracic approach may offer better clinical effect of short-term postoperative recovery.

\section{Acknowledgments}

Funding: None.

\section{Footnote}

Reporting Checklist: The authors have completed the STROBE reporting checklist. Available at http://dx.doi. org/10.21037/jtd-20-266 
Data Sharing Statement: Available at http://dx.doi. org/10.21037/jtd-20-266

Peer Review File: Available at http://dx.doi.org/10.21037/jtd20-266

Conflicts of Interest: All authors have completed the ICMJE uniform disclosure form (available at http://dx.doi. org/10.21037/jtd-20-266). JH serves as an unpaid Executive Editor-in-Chief of Fournal of Thoracic Disease. WL serves as an unpaid editorial board member of fournal of Thoracic Disease. The other authors have no conflicts of interest to declare.

Ethical Statement: The authors are accountable for all aspects of the work in ensuring that questions related to the accuracy or integrity of any part of the work are appropriately investigated and resolved. The study was conducted in accordance with the Declaration of Helsinki (as revised in 2013) and the Harmonized Tripartite Guideline for Good Clinical Practice from the International Conference on Harmonization. The study protocol and methods were reviewed by the institutional ethics committee of hospital. The ID/number of ethics approval of this study is (2019-SVA). Informed and written consents for each patient were obtained.

Open Access Statement: This is an Open Access article distributed in accordance with the Creative Commons Attribution-NonCommercial-NoDerivs 4.0 International License (CC BY-NC-ND 4.0), which permits the noncommercial replication and distribution of the article with the strict proviso that no changes or edits are made and the original work is properly cited (including links to both the formal publication through the relevant DOI and the license). See: https://creativecommons.org/licenses/by-nc-nd/4.0/.

\section{References}

1. Bacha EA, Chapelier AR, Macchiarini P, et al. Surgery for invasive primary mediastinal tumors. Ann Thorac Surg 1998;66:234-9.

2. Wu S, Liang H, Liang W, et al. Single- versus two-port video-assisted thoracic surgery in mediastinal tumor: a propensity-matched study. J Thorac Dis 2019;11:4428-35.

3. Kitagawa H, Namikawa T, Iwabu J, et al. Comparison between neck-first approach and thoracic approach during thoracoscopic esophagectomy. Langenbecks Arch Surg
2017;402:1159-65.

4. Hwang SK, Park SI, Kim YH, et al. Clinical results of surgical resection of mediastinal teratoma: efficacy of video-assisted thoracic surgery. Surg Endosc 2016;30:4065-8.

5. Nagano H, Suda T, Ishizawa H, et al. Video-assisted thoracoscopic surgery for ectopic mediastinal parathyroid tumor: subxiphoid and lateral thoracic approach. J Thorac Dis 2019;11:2932-8.

6. Ye B, Tantai JC, Ge XX, et al. Surgical techniques for early-stage thymoma: video-assisted thoracoscopic thymectomy versus transsternal thymectomy. J Thorac Cardiovasc Surg 2014;147:1599-603.

7. Da M, Peng W, Mo X, et al. Comparison of efficacy between video-assisted thoracoscopic surgery and thoracotomy in children with mediastinal tumors: 6-year experience. Ann Transl Med 2019;7:653.

8. Abu-Akar F, Gonzalez-Rivas D, Yang C, et al. Subxiphoid Uniportal VATS for Thymic and Combined Mediastinal and Pulmonary Resections - A Two-Year Experience. Semin Thorac Cardiovasc Surg 2019;31:614-9.

9. Detterbeck FC. Clinical value of the WHO classification system of thymoma. Ann Thorac Surg 2006;81:2328-34.

10. Agatsuma H, Yoshida K, Yoshino I, et al. Video-Assisted Thoracic Surgery Thymectomy Versus Sternotomy Thymectomy in Patients With Thymoma. Ann Thorac Surg 2017;104:1047-53.

11. Pennathur A, Qureshi I, Schuchert MJ, et al. Comparison of surgical techniques for early-stage thymoma: feasibility of minimally invasive thymectomy and comparison with open resection. J Thorac Cardiovasc Surg 2011;141:694-701.

12. von Elm E, Altman DG, Egger M, et al. The Strengthening the Reporting of Observational Studies in Epidemiology (STROBE) statement: guidelines for reporting observational studies. PLoS Med 2007;4:e296.

13. Wu CY, Heish MJ, Wu CF. Single port VATS mediastinal tumor resection: Taiwan experience. Ann Cardiothorac Surg 2016;5:107-11.

14. Jiang L, Depypere L, Rocco G, et al. Spontaneous ventilation thoracoscopic thymectomy without muscle relaxant for myasthenia gravis: Comparison with "standard" thoracoscopic thymectomy. J Thorac Cardiovasc Surg 2018;155:1882-9.e3.

15. Hsu CP. Subxiphoid approach for thoracoscopic thymectomy. Surg Endosc 2002;16:1105.

16. Liu YW, Chen HW, Lee JY, et al. Is a Chest Tube Necessary after Video-Assisted Thoracoscopic Mediastinal Tumor Resection? Thorac Cardiovasc Surg 2019. [Epub 
ahead of print].

17. Petersen RH, Holbek BL, Hansen HJ, et al. Video-assisted thoracoscopic surgery-taking a step into the future. Eur J Cardiothorac Surg 2017;51:694-5.

18. Arom KV, Franz JL, Grover FL, et al. Subxiphoid anterior mediastinal exploration. Ann Thorac Surg 1977;24:289-90.

19. Cai H, Xie D, Al Sawalhi S, et al. Subxiphoid versus intercostal uniportal video-assisted thoracoscopic surgery for bilateral lung resections: a single-institution experience. Eur J Cardiothorac Surg 2020;57:343-9.

20. Zhang L, Li M, Jiang F, et al. Subxiphoid versus lateral intercostal approaches thoracoscopic thymectomy for non-myasthenic early-stage thymoma: A propensity score -matched analysis. Int J Surg 2019;67:13-7.

21. Wu N, Wu L, Qiu C, et al. A comparison of video-assisted thoracoscopic surgery with open thoracotomy for the management of chest trauma: a systematic review and meta-analysis. World J Surg 2015;39:940-52.

22. Oh DS, Reddy RM, Gorrepati ML, et al. Robotic-Assisted, Video-Assisted Thoracoscopic and Open Lobectomy: Propensity-Matched Analysis of Recent Premier Data.

Cite this article as: Mao Y, Lan Y, Cui F, Deng H, Zhang Y, Wu X, Liang W, Liu J, Liang H, He J. Comparison of different surgical approaches for anterior mediastinal tumor. J Thorac Dis 2020;12(10):5430-5439. doi: 10.21037/jtd-20-266
Ann Thorac Surg 2017;104:1733-40.

23. Qian L, Chen X, Huang J, et al. A comparison of three approaches for the treatment of early-stage thymomas: robot-assisted thoracic surgery, video-assisted thoracic surgery, and median sternotomy. J Thorac Dis 2017;9:1997-2005.

24. Duan X, Shang X, Tang P, et al. Lymph node dissection for Siewert II esophagogastric junction adenocarcinoma: a retrospective study of 136 cases. ANZ J Surg 2018;88:E264-E267.

25. Allakhverdiev A, Davydov M, Allakhverdieva G, et al. Thoracoscopic thymectomy - The method of choise in surgical treatment of non-invasive thymomas. Ann Med Surg (Lond) 2019;42:29-34.

26. Sehitogullari A, Nasir A, Anbar R, et al. Comparison of perioperative outcomes of videothoracoscopy and robotic surgical techniques in thymoma. Asian J Surg 2020;43:244-50.

27. Efteev LA, Rodionov EO, Miller SV, et al. Thoracoscopy in combined treatment of thymoma (in Russian only). Khirurgiia (Mosk) 2019;(3):84-7. 